\title{
Ärztliches Vorwort
}

\section{Hon.-Prof. (DPU) Dr. med. Günther Jonitz Präsident der Ärztekammer Berlin a. D.}

Sehr verehrte Frau Kollegin, sehr geehrter Herr Kollege, die wesentliche Aufgabe des Arztes und der Ärztin besteht darin, Krankheiten zu erkennen und zu heilen, Beschwerden zu lindern, Leben zu verlängern und Patientinnen und Patienten dabei zu unterstützen, trotz gesundheitlicher Einschränkung ein möglichst selbstbestimmtes Leben führen zu können. Ärztinnen und Ärzte sind dabei ausschließlich ihrem Gewissen verantwortlich und den Regeln der ärztlichen Kunst unterworfen.

Dazu gehören nicht nur die klassischen klinisch-ärztlichen Kompetenzen, sondern auch die der Begutachtung. Um ein selbstbestimmtes Leben zu führen, ist die Feststellung des medizinischen Zustandes mit seinen Auswirkungen auf den gesundheitlichen Zustand, deren Ursachen und Folgen, oft von zentraler Bedeutung. Von der Klärung dieser Fragen hängt oft nicht nur die materielle, sondern auch die emotionale Lebensgrundlage ab. Auch bei der Begutachtung gelten die Regeln der ärztlichen Kunst, sorgfältig, mit Expertise und wissenschaftsbasiert zu handeln. Dies zu unterstützen dient dieses Buch.

Das Buch bezieht sich auch auf das Curriculum „Medizinische Begutachtung“ der Bundesärztekammer. Ziel des Curriculums und damit auch dieses Buches ist eine grundlegende Fortbildung von Ärztinnen und Ärzten, die sich gutachterlichen Aufgaben annehmen wollen. Dabei werden neben umfassenden Kenntnissen zur medizinischen Begutachtung die Unterschiede zwischen Kausalitäts- und Zustandsgutachten in den verschiedenen Rechtsgebieten des Straf-, Zivil- und Sozialrechts behandelt. Wichtige formale Kriterien der Gutachtenerstellung und der gutachterlichen Aussage werden ebenso vermittelt wie spezifische Aspekte aus unterschiedlichen Fachdisziplinen der Medizin.

Doch nicht nur für die Patientinnen und Patienten ist die Begutachtung wichtig. Auch für Ärztinnen und Ärzte stellt sie eine oft bereichernde Erweiterung ihrer präventiven, kurativen und palliativen ärztlichen Tätigkeit dar. Sie ist mit einem Rollenwechsel vom „Behandler“ zum „Begutachter“ verbunden, der bewusst vollzogen und gelebt werden muss. Vom Berater eines Gerichts oder Auftragnehmer einer Streitpartei ist ärztlicher Sachverstand gefordert. Dieser ist nach wissenschaftlichen und evidenzbasierten Kriterien vorurteilsfrei und neutral anzuwenden und anderen Professionen in verständlicher Ausdrucksweise darzulegen. Der oder die medizinisch Begutachtende ist somit nicht nur einer Patientin oder einem Patienten verpflichtet, sondern leistet gesamtgesellschaftlich einen Beitrag zur wissenschaftlich begründeten Rechtsfindung und Rechtsprechung. 
VI — Ärztliches Vorwort Prof. Dr. Günther Jonitz

Ich danke den Herausgebern und Beitragenden für dieses wegweisende Buch, das bereits jetzt die Grundlagen eines Standardwerkes für dieses wichtige Thema aufweist.

Mit freundlichen kollegialen Grüßen

Ihr Günther Jonitz 